\title{
POSITIVE HARMONIC FUNCTIONS VANISHING ON THE BOUNDARY FOR THE LAPLACIAN IN UNBOUNDED HORN-SHAPED DOMAINS
}

\author{
DIMITRY IOFFE AND ROSS PINSKY
}

\begin{abstract}
Denote points $\bar{x} \in R^{d+1}, d \geq 2$, by $\bar{x}=(\rho, \theta, z)$, where $\rho>0$, $\theta \in S^{d-1}$, and $z \in R$. Let $a:[0, \infty) \rightarrow(0, \infty)$ be a nondecreasing $C^{2}-$ function and define the "horn-shaped" domain $\Omega=\{\bar{x}=(\rho, \theta, z):|z|<$ $a(\rho)\}$ and its unit "cylinder" $D=\{\bar{x}=(\rho, \theta, z) \in \Omega: \rho<1\}$. Under appropriate regularity conditions on $a$, we prove the following theorem: (i) If $\int^{\infty} a(\rho) / \rho^{2} d \rho=\infty$, then the Martin boundary at infinity for $\frac{1}{2} \Delta$ in $\Omega$ is a single point. (ii) If $\int^{\infty} a(\rho) / \rho^{2} d \rho<\infty$, then the Martin boundary at infinity for $\frac{1}{2} \Delta$ in $\Omega$ is homeomorphic to $S^{d-1}$. More specifically, a sequence $\left\{\left(\rho_{n}, \theta_{n}, z_{n}\right)\right\}_{n=1}^{\infty} \subset \Omega$ satisfying $\lim _{n \rightarrow \infty} \rho_{n}=\infty$ is a Martin sequence if and only if $\lim _{n \rightarrow \infty} \theta_{n}$ exists on $S^{d-1}$. From (i), it follows that the cone of positive harmonic functions in $\Omega$ vanishing continuously on $\partial \Omega$ is onedimensional. From (ii), it follows easily that the cone of positive harmonic functions on $\Omega$ vanishing continuously on $\partial \Omega$ is generated by a collection of minimal elements which is homeomorphic to $S^{d-1}$.

In particular, the above result solves a problem stated by Kesten, who asked what the Martin boundary is for $\frac{1}{2} \Delta$ in $\Omega$ in the case $a(\rho)=1+\rho^{\gamma}, 0<\gamma<$ 1 . Our method of proof involves an analysis as $\rho \rightarrow \infty$ of the exit distribution on $\partial D$ for Brownian motion starting from $(\rho, \theta, z) \in \Omega$ and conditioned to hit $D$ before exiting $\Omega$.
\end{abstract}

Denote points $\bar{x} \in R^{d+1}, d \geq 2$, by $\bar{x}=(x, z)$, where $x \in R^{d}$ and $z \in R^{1}$. Consider $\frac{1}{2} \Delta$ in the "horn-shaped" domain $\Omega=\{\bar{x}=(x, z) \in$ $\left.R^{d+1}:|z|<a(|x|)\right\}$, where $a:[0, \infty) \rightarrow(0, \infty)$ is a $C^{2}$-function. Since $\partial \Omega$ is a smooth boundary (Lipschitz would be enough), it follows from a result of Hunt and Wheeden [3] that every Euclidean boundary point on $\partial \Omega$ may be identified with a distinct Martin boundary point. Thus, the interesting part of the Martin boundary for $\frac{1}{2} \Delta$ in $\Omega$ is the "Martin boundary at infinity", that is, the part of the Martin boundary arising from sequences $\left\{\bar{x}_{n}\right\}_{n=1}^{\infty} \subset \Omega$ satisfying $\lim _{n \rightarrow \infty}\left|\bar{x}_{n}\right|=\infty$, or, in terms of positive harmonic functions, the part of the Martin boundary which corresponds to positive harmonic functions on $\Omega$ which vanish on $\partial \Omega$. We will give a probabilistic proof of the following theorem, the motivation and intuition for which is discussed below.

Received by the editors June 26, 1992.

1991 Mathematics Subject Classification. Primary 35J05; Secondary 60J50, 31C35, 31B05, $35 \mathrm{~J} 15$. 
Theorem 1. Let $\Omega=\left\{\bar{x}=(x, z) \in R^{d+1}:|z|<a(|x|)\right\}$, where $0<a \in$ $C^{2}([0 \infty))$ satisfies

(i) $a^{\prime} \geq 0$;

(ii) $a(\rho) / \rho$ is nonincreasing,

(iii) $a^{\prime \prime} \leq 0$;

(iv) the curvature $k(\rho) \equiv-a^{\prime \prime}(\rho) /\left(1+\left(a^{\prime}(\rho)\right)^{2}\right)^{3 / 2}$ is nonincreasing and satisfies $\lim _{\rho \rightarrow \infty} a(\rho) k(\rho)=0$.

Let $\rho=|x|$ and $\theta=x /|x| \in S^{d-1}$ denote polar coordinates for $x \in R^{d}$.

(a) If $\int^{\infty} a(\rho) / \rho^{2} d \rho=\infty$, then the Martin boundary at infinity for $\frac{1}{2} \Delta$ in $\Omega$ consists of a single point.

(b) If $\int^{\infty} a(\rho) / \rho^{2} d \rho<\infty$, then the Martin boundary at infinity for $\frac{1}{2} \Delta$ in $\Omega$ is isomorphic to $S^{d-1}$. More specifically, a sequence $\left\{\left(\rho_{n}, \theta_{n}, z_{n}\right)\right\}_{n=1}^{\infty}$ $\subset \Omega$ satisfying $\lim _{n \rightarrow \infty} \rho_{n}=\infty$ is a Martin sequence if and only if $\lim _{n \rightarrow \infty} \theta_{n}$ exists in $S^{d-1}$.

Remark 1. The statement of the theorem is actually a refined version of an open problem suggested by Harry Kesten [5]. Kesten suggested studying the case $a(\rho)=1+\rho^{\gamma}$, with $0<\gamma<1$. We thank John Taylor for bringing this to our attention.

Remark 2. The conditions on $a$ allow, for example, $a(\rho)=\rho^{\gamma}+1$, with $0 \leq$ $\gamma \leq 1$, or $a(\rho)=\rho^{\gamma} /(\log (\rho+2))^{\delta}+1$, with $0<\gamma<1$ and $-\infty<\delta<\infty$, with $\gamma=0$ and $\delta \leq 0$ or with $\gamma=1$ and $\delta \geq 0$. In particular, if $a(\rho)=\rho^{\gamma}+1$, then the Martin boundary at infinity is isomorphic to $S^{d-1}$ if $0 \leq \gamma<1$, and is a single point if $\gamma=1$. If $a(\rho)=\rho /(\log (\rho+2))^{\delta}+1$, then the Martin boundary at infinity is isomorphic to $S^{d-1}$ if $\delta>1$ and is a single point if $0 \leq \delta \leq 1$.

Remark 3. It is easy to see that, due to symmetry considerations, in case (b) every Martin boundary point at infinity is a minimal Martin boundary point. Thus, in case (a) there is exactly one minimal positive harmonic function in $\Omega$ which vanishes on $\partial \Omega$, while in case (b) the class of such functions is isomorphic to $S^{d-1}$.

Remark 4. In fact, we know how to amend the proofs to cover the case in which $a^{\prime} \leq 0, a^{\prime \prime} \geq 0, a(\rho) / \rho$ is nonincreasing, $\inf _{\rho \geq 0} a(\rho) / a(\delta \rho) \geq c \delta$, for all $0<\delta<1$ and some $c>0$, and a curvature condition similar to condition (iv) is imposed. This would allow, for example, $a(\rho)=(\rho+1)^{\gamma}$, with $-1 \leq \gamma<0$.

Remark 5. Conditions (i) and (ii) in fact follow automatically from condition (iii) and the positivity of $a$; they have been expressed explicitly in the statement of the theorem because they will be referred to frequently in the sequel.

The probabilistic connection to Theorem 1 is as follows. Let $D=\{\bar{x}=$ $(x, z) \in \Omega:|x|<1\}$ denote the unit "cylinder" in $\Omega$. Let $P_{\bar{x}}$ denote $(d+$ 1)-dimensional Wiener measure centered at $\bar{x} \in \Omega$ and let $X(t)$ denote the corresponding Brownian motion. Define $\tau_{\Omega}=\inf \{t \geq 0: X(t) \notin \Omega\}$ and $\sigma_{D}=\inf \{t \geq 0: X(t) \in \bar{D}\}$. Let $h(\bar{x})=P_{\bar{x}}\left(\sigma_{D}<\tau_{\Omega}\right)$ and define the $h$ transformed measure $P_{\bar{x}}^{h}$ by

$$
P_{\bar{x}}^{h}\left(\left\{X(t), 0 \leq t \leq \sigma_{D}\right\} \in \cdot\right)=P_{\bar{x}}\left(\left\{X(t), 0 \leq t \leq \sigma_{D}\right\} \in \cdot \mid \sigma_{D}<\tau_{\Omega}\right) .
$$


That is, $P_{\bar{x}}^{h}$ is the measure corresponding to Brownian motion in $\Omega$ conditioned to hit $\bar{D}$ before exiting $\Omega$. As is well known [2], $P h$ is the diffusion measure corresponding to the $h$-transformed operator $\frac{1}{2} \Delta^{h}$ defined by $\frac{1}{2} \Delta^{h} u=\frac{1}{h} \cdot \frac{1}{2} \Delta(h u)$. Define the harmonic probability measure $\mu_{\frac{h}{x}}$ on $\partial D$ by

$$
\mu_{\bar{x}}^{h}(d y)=P_{\bar{x}}\left(X\left(\sigma_{D}\right) \in d y\right)=P_{\bar{x}}\left(X\left(\sigma_{D}\right) \in d y \mid \sigma_{D}<\tau_{\Omega}\right), \quad \text { for } \bar{x} \in \Omega-D .
$$

The following theorem, which is a special case of [7, Theorem 1], relates the Martin boundary for $\frac{1}{2} \Delta$ in $\Omega$ to the harmonic measures $\mu_{\bar{x}}^{h}$.

Theorem A. Let $\left\{\bar{x}_{n}\right\}_{n=1}^{\infty} \subset \Omega-D$ satisfy $\lim _{n \rightarrow \infty}\left|\bar{x}_{n}\right|=\infty$. Then $\left\{\bar{x}_{n}\right\}_{n=1}^{\infty}$ is a Martin sequence if and only if $\mathrm{w}-\lim _{n \rightarrow \infty} \mu_{\bar{x}_{n}}^{h}$ exists.

Remark. [7, Theorem 1] actually deals with more general operators than $\frac{1}{2} \Delta$, but the domains $\Omega$ and $D$ above are replaced respectively by $R^{d}$ and any compact domain with a smooth boundary. One can check that the same proof goes through with $\Omega$ and $D$. (See [8, Proposition 8.7.1] for a similar result.)

We will prove the following theorem.

Theorem 2. Let $\Omega=\left\{\bar{x}=(x, z) \in R^{d+1}:|z|<a(|x|)\right.$, where $a:[0, \infty) \rightarrow$ $(0, \infty)$ satisfies

(i) $a^{\prime} \geq 0$,

(ii) $a(\rho) / \rho$ is nonincreasing,

(iii) $a^{\prime \prime} \leq 0$,

(iv) The curvature $k(\rho) \equiv-a^{\prime \prime}(\rho) /\left(1+\left(a^{\prime}(\rho)\right)^{2}\right)^{3 / 2}$ is nonincreasing and satisfies $\lim _{\rho \rightarrow \infty} a(\rho) k(\rho)=0$.

Let $(\rho, \theta)$ denote the spherical coordinates for $x \in R^{d}$ and let $\left\{\bar{x}_{n}\right\}_{n=1}^{\infty}=$ $\left\{\left(\rho_{n}, \theta_{n}, z_{n}\right)\right\}_{n=1}^{\infty} \subset \Omega$ satisfy $\lim _{n \rightarrow \infty} \rho_{n}=\infty$.

(a) If $\int^{\infty} a(\rho) / \rho^{2} d \rho=\infty$, then $\mathrm{w}-\lim _{n \rightarrow \infty} \mu_{\left(\rho_{n}, \theta_{n}, z_{n}\right)}$ exists.

(b) If $\int^{\infty} a(\rho) / \rho^{2} d \rho<\infty$, then $\mathrm{w}-\lim _{n \rightarrow \infty} \mu_{\left(\rho_{n}, \theta_{n}, z_{n}\right)}^{h}$ exists if and only if $\lim _{n \rightarrow \infty} \theta_{n}$ exists in $S^{d-1}$.

Theorem 1 follows immediately from Theorem A and Theorem 2 .

In the case $a(\rho) \equiv c$, the domain $\Omega$ is a strip. The case of a general strip of the form $R^{d} \times A$, where $A \subset R^{m}$ is a bounded domain with a smooth boundary, is treated in [8, Theorem 8.6.6 and Proposition 8.8.1]. (The case of a general strip of the form $R^{d} \times A$, where $A \subset R^{m}$ is a bounded domain with a Lipschitz boundary, has been treated by analytic methods in [9].) Restricting to the present strip for the sake of exposition, what is shown in [8] is that w$\lim _{n \rightarrow \infty} \mu_{\rho_{n}, \theta_{n}, z_{n}}^{h}$ converges if and only if $\lim _{n \rightarrow \infty} \theta_{n}$ exists on $S^{d-1}$. That is, as the initial point $\bar{x}_{n}=\left(\rho_{n}, \theta_{n}, z_{n}\right) \in \Omega$ approaches infinity, the harmonic measure on the boundary $\partial D$ of the cylinder $D$ for Brownian motion starting from $\bar{x}_{n}$ and conditioned to hit $\partial D$ before exiting the strip $\Omega$ is such that its angular component $d \theta$ does not ergodize but its $d z$-component does ergodize. As can be seen clearly in [8], the reason for this is that the conditioning introduces a drift, bounded between two negative constants, into the $\rho$-component of the process. Therefore, the expected time it takes the conditioned Brownian motion to reach $\partial D$ starting from $x \in \Omega$ is on the order $|x|$ for large $|x|$. For 
classical diffusion, this order would be, of course, $|x|^{2}$ and in fact this is essentially the minimal order necessary for the $\theta$-component to ergodize. Thus, the motivation and intuition for Theorem 1 is as follows. The larger the function $a$ is, the less of an effect the conditioning ought to have. The angular component should ergodize only if the conditioning is so minimal that the conditioned Brownian motion behaves over large distances more or less like unconditioned Brownian motion. Theorem 2 then identifies the critical growth rate of $a$.

Consider now for a moment the situation in which $\lim _{\rho \rightarrow \infty} a(\rho)=0$. Remark 4 following Theorem 1 indicates that, at least under the condition imposed there, the Martin boundary is still isomorphic to $S^{d-1}$. The condition in Remark 4 allows for decay on an order no faster than $1 / \rho$. Regardless of how fast $a(\cdot)$ decays, one still expects, of course, that the $\theta$-component will not ergodize. However, if $a(\cdot)$ decays sufficiently fast, the conditioned Brownian motion will be catapulted inward from infinity in finite time, which may lead one to suspect that the $z$-component will no longer ergodize. On the other hand, the fact that $z$ is confined to such a narrow range for large $\rho$ might allow for a kind of "instantaneous" ergodization at infinity thereby rendering the finite entrance time from infinity irrelevant. We suspect that the $z$-component will ergodize no matter how fast $a(\cdot)$ decays.

The conditions imposed on the function $a(\cdot)$ in Theorems 1 and 2 prevent much local fluctuation and also prevent superlinear growth. However, the probabilistic intuition above suggests that such restrictions should not be necessary. Unfortunately, a comparison of probabilities with different $h$-transforms requires comparing ratios of probabilities and this is usually quite difficult. We make the following conjecture.

Conjecture. Let $\Omega=\left\{\bar{x}=(x, z) \in R^{d+1}:|z|<a(|x|)\right\}$, where $a:[0, \infty) \rightarrow$ $(0, \infty)$ is continuous and let $\Omega_{i}, i=1,2$, be two domains satisfying the conditions of Theorem 1 .

(i) If the Martin boundaries at infinity for $\Omega_{1}$ and $\Omega_{2}$ are both isomorphic to $S^{d-1}$, and $\Omega_{1} \subset \Omega \subset \Omega_{2}$, then the Martin boundary at infinity for $\Omega$ is also isomorphic to $S^{d-1}$.

(ii) If the Martin boundaries at infinity for $\Omega_{1}$ and $\Omega_{2}$ are both single points, and $\Omega_{1} \subset \Omega \subset \Omega_{2}$, then the Martin boundary for $\Omega$ is also a single point.

(iii) If the Martin boundary at infinity for $\Omega_{1}$ is a single point, and $\Omega_{1} \subset \Omega$, then the Martin boundary at infinity for $\Omega$ is also a single point.

(iv) If the Martin boundary at infinity for $\Omega_{1}$ is isomorphic to $S^{d-1}$ and $\Omega \subset \Omega_{1}$, then the Martin boundary at infinity for $\Omega$ is also isomorphic to $S^{d-1}$.

More ambitiously, perhaps the sandwiching is unnecessary and the integral condition determines the Martin boundary at infinity for all domains $\Omega$ of the form specified in the conjecture. As additional support for these conjectures which state, more or less, that local boundary fluctuations do not affect the Martin boundary at infinity, consider domains in $R^{2}$ of the form $\Omega=\{(x, y) \in$ $\left.R^{2}: a(x)<y<b(x)\right\}$, where $a(x)<b(x)$. If $a(x) \equiv 0$ and $b(x) \equiv 1$, then the Martin boundary at infinity consists of two points [8, Theorem 8.6.6]. Thus, an application of the Riemann mapping theorem shows that the minimal Martin 
boundary at infinity must be two points for any domain of the form $\Omega$.

In $\S 2$, we prove Theorem 2 . The strategy is as follows. It is quite untenable to work directly with $\Omega-D$, the horn-shaped domain minus its "unit cylinder". Thus, we make a change of variables to map $\Omega-D$ to a strip minus its unit cylinder. The change of variables is of the form $r=r(\rho, z)$, with $r(1, z)=1$ and $r_{\rho}>0$, and $w=w(\rho, z)$, with $w(\rho, \pm a(\rho))= \pm 1$ and $w_{z}>0$; the $\theta$-variable is left untouched. The image of $\Omega-\bar{D}$ is

$$
\Sigma \equiv\left\{(r, \theta, w): r>1, \theta \in S^{d-1},|w|<1\right\} .
$$

Of course, this domain simplification exacts a price-the Laplacian must be replaced by a more general second-order elliptic operator. By making a judicious choice for the change of variables ( $r$ and $w$ are chosen to be conjugate harmonic functions), the principal part of the operator which replaces the Laplacian will still be in a relatively simple form. After dividing out by a certain scalar, this operator is given by (2.1). We now consider the diffusion on $\Sigma$ corresponding to $L$ as in (2.1) and conditioned to reach $\{r=1\}$ before reaching $\{|w|=1\}$. By Doob's theory, this conditioned diffusion corresponds to an $h$-transformed operator, $L^{h_{0}}$, where $h_{0}=h_{0}(r, w)$ is an appropriate $L$-harmonic function. Let $\nu_{r, \theta, w}^{h_{0}}$ denote the harmonic probability measure on $\{r=1\}$ corresponding to the condition diffusion starting from $(r, \theta, w) \in \Sigma$. To prove Theorem 2, we must study the behavior of $\nu_{r, \theta, w}^{h_{0}}$ as $r \rightarrow \infty$. By (2.1), it follows that the $\theta$-component of the conditioned diffusion may be represented in skew-product form; thus $\nu_{r, \theta, w}^{h_{0}}$ has a skew-product decomposition. Using a blend of analytical and probabilistic arguments, the coefficients of $L$ in (2.1) can be estimated in terms of $a(\cdot)$. Using the skew-product representation and the estimates on the coefficients of $L$ in terms of $a(\cdot)$, we are able to relate the behavior of $\nu_{r, \theta, w}^{h_{0}}$ as $r \rightarrow \infty$ to the behavior of $a(\cdot)$. Most of the "hard" work is done in Lemmas 1 and 5. In particular, the methods used in the proof of Lemma 1 may be of some independent interest and may be useful with regard to some related problems.

\section{PROOF OF THEOREM 2}

We begin with a change of variables that will reduce the geometry to a strip. Let $w(\rho, z)$ denote the solution to $\frac{1}{2}\left(w_{\rho \rho}+w_{z z}\right)=0$ in $\{(\rho, z): \rho>1$, $|z|<a(\rho)\}$, with $w(\rho, \pm a(\rho))= \pm 1$, for $\rho>1$, and $w_{\rho}(1, z)=0$, for $|z|<a(1)$. We make the change of variables $(\rho, \theta, z) \rightarrow(r, \theta, w)$, where $r=1$ on $\rho=1$ and $d r=w_{z} d \rho-w_{\rho} d z$. In the new variables, the domain $\Omega-\bar{D}$ takes the form $\Sigma$; that is, $\Sigma$ is a strip minus its unit cylinder. In $(r, \theta, w)$-coordinates, $\frac{1}{2} \Delta$ takes the form (after division by $\left.|\nabla w|^{2}\right)$

$$
\begin{aligned}
L \equiv & \frac{1}{2} \frac{\partial^{2}}{\partial r^{2}}+\frac{1}{2} \frac{\partial^{2}}{\partial w^{2}}+\frac{(d-1) w_{z}}{2 \rho|\nabla w|^{2}(\rho, z)} \frac{\partial}{\partial r} \\
& +\frac{w_{\rho}(\rho, z)}{2 \rho|\nabla w|^{2}(\rho, z)} \frac{\partial}{\partial w}+\frac{1}{2 \rho^{2}|\nabla w|^{2}(\rho, z)} \Delta_{S^{d-1}},
\end{aligned}
$$

where $\Delta_{S^{d-1}}$ is the Laplace-Beltrami operator on $S^{d-1}$.

The following lemma will play a crucial role. 
Lemma 1. There exist constants $c_{1}, c_{2}>0$ such that for $\rho>1$ and $|z|<a(\rho)$,

(i) $c_{1} / a(\rho) \leq w_{z}(\rho, z) \leq c_{2} / a(\rho)$, and

(ii) $\left|w_{\rho}(\rho, z)\right| \leq c_{2} / a(\rho)$.

Proof. Let $n$ denote the outward unit normal vector of $\{(\rho, z):|z|<a(\rho)\}$ at $|z|=a(\rho)$. We begin by showing that there exist constants $\gamma_{1}, \gamma_{2}>0$ such that

$$
\gamma_{1} / a(\rho) \leq \frac{\partial w}{\partial n}(\rho, z) \leq \gamma_{2} / a(\rho), \quad \text { for } \rho>1 \text { and } z=a(\rho) .
$$

By the Hopf maximum principle, $\partial w / \partial n>0$; thus it is in fact enough to prove (2.2) for large $\rho$. Let $\rho_{0}>1$ and define $x_{0}=\left(\rho_{0}, a\left(\rho_{0}\right)\right)$.

We consider first the upper bound in (2.2). By conditions (i) and (iii) (or by condition (ii)) on $a(\rho)$ in Theorem 2, we have $\sup _{\rho \geq 1} a^{\prime}(\rho)<\infty$. Using condition (ii), a simple calculation reveals that we may choose a $c \in(0,1]$ such that, for all large $\rho_{0}$, the $\rho$-component of $x_{0}+c a\left(\rho_{0}\right) n\left(x_{0}\right)$ is larger than 1 . Let $x_{m}=x_{0}+c a\left(\rho_{0}\right) n\left(x_{0}\right)$. We may also choose a $\gamma>0$ such that the ball $B \equiv B\left(x_{m},(c+\gamma) a\left(\rho_{0}\right)\right)$ of radius $(c+\gamma) a\left(\rho_{0}\right)$ centered at $x_{m}$ lies completely in $\{(\rho, z): z>0\}$. Let $G=B \cap\{(\rho, z): \rho>1$ and $z<a(\rho)\}$. Define $v(x)=w(x) \log (1+\gamma / c)+\log \left|x-x_{m}\right|$, for $x \in \bar{G}$, and note that $v$ is harmonic in $G$ for the operator $\frac{1}{2}\left(\partial^{2} / \partial \rho^{2}+\partial^{2} / \partial z^{2}\right)$. Thus, by the maximum principle, $v$ attains its minimum in $\bar{G}$ at a point on $\partial G$. Now $\partial G \cap\{\rho=1\}$ may or may not be empty; however, in any case, $v$ cannot attain its minimum on $\bar{G}$ at a point in $\partial G \cap\{\rho=1\}$. Indeed, since the $\rho$-component of $x_{m}$ is larger than 1 , it follows that $\partial v / \partial \rho<0$ on $\partial G \cap\{\rho=1\}$. By symmetry, $w(\rho, z) \geq 0$ if $z \geq 0$. By condition (iii) on $a(\rho)$ in Theorem 2, $a(\rho)$ is concave; thus $\left|x-x_{m}\right|$ on $\{z=a(\rho)\}$ attains its minimum uniquely at $x_{0}$. Therefore, since $\partial G \cap\{z<0\}=\varnothing$, it is easy to see that $v$ restricted to $\partial G \cap\{\rho>1\}$ attains its minimum at $x_{0}$. We conclude from these considerations that $v$ attains its minimum on $\bar{G}$ at $x_{0}$. Thus $\partial v / \partial n\left(x_{0}\right) \leq 0$ or, equivalently, $(\log (1+\gamma / c)) \partial w / \partial n\left(x_{0}\right) \leq 1 / c a\left(\rho_{0}\right)$. This proves the upper bound in (2.2).

We now turn to the lower bound in inequality (2.2). This time, let $x_{m}=$ $x_{0}-\frac{3}{2} a\left(\rho_{0}\right)\left(1+\left(a^{\prime}\left(\rho_{0}\right)\right)^{2}\right)^{1 / 2} h\left(x_{0}\right)$ and let $B \equiv B\left(x_{m}, \frac{3}{2} a\left(\rho_{0}\right)\left(1+\left(a^{\prime}\left(\rho_{0}\right)\right)^{2}\right)^{1 / 2}\right)$. We first show that, for large $\rho_{0}, B \cap\{\rho \leq 2\}=\varnothing$. A simple calculation reveals that

$$
\inf \{\rho:(\rho, z) \in B\}=\rho_{0}+\frac{3}{2} a\left(\rho_{0}\right) a^{\prime}\left(\rho_{0}\right)-\frac{3}{2} a\left(\rho_{0}\right)\left(1+\left(a^{\prime}\left(\rho_{0}\right)\right)^{2}\right)^{1 / 2} .
$$

By conditions (i) and (iii) on $a(\rho)$ in Theorem $2, \lim _{\rho \rightarrow \infty} a^{\prime}(\rho)$ exists, and by condition (ii), $\lim _{\rho \rightarrow \infty} a(\rho) / \rho$ exists. A little calculus then shows in fact that for large $\rho_{0}, \inf \{\rho:(\rho, z) \in B\}>\frac{1}{4} \rho_{0}$.

We now show that the curve $\{z=a(\rho), \rho \geq 1\}$ intersects $\partial B$ only at $x_{0}$. Recall that the radius of curvature $R\left(\rho_{0}\right)$ is given by $R\left(\rho_{0}\right)=1 / k\left(\rho_{0}\right)$. By condition (iv) on $a(\rho)$ in Theorem 2, for large $\rho_{0}$, the radius of $B$ is less than the radius of curvature $R\left(\rho_{0}\right)$. Therefore, in a neighborhood of $x_{0}$, the curve lies above $\partial B$. Now assume that $\{z=a(\rho), \rho \geq 1\} \cap \partial B \cap\left\{\rho>\rho_{0}\right\} \neq$ $\varnothing$ and let $x_{1} \equiv\left(\rho_{1}, a\left(\rho_{1}\right)\right)$ denote the point in that intersection whose $\rho$ coordiante is the smallest. Then at some point on the curve between $x_{0}$ and $x_{1}$, the curvature must be larger than the curvature of $\partial B$. But the curvature of $\partial B$ is the reciprocal of its radius and, thus, is larger than $k\left(\rho_{0}\right)$. This would 
contradict the monotonicity property of the curvature in condition (iv). Now assume that $\{z=a(\rho), \rho \geq 1\} \cap \partial B \cap\left\{\rho<\rho_{0}\right\} \neq \varnothing$ and let $x_{1} \equiv\left(\rho_{1}, a\left(\rho_{1}\right)\right)$ denote the point in that intersection whose $\rho$-coordinate is the largest. Since $B \cap\{\rho \leq 2\}=\varnothing$, the above intersection must contain at least two points; let $x_{2} \equiv\left(\rho_{2}, a\left(\rho_{2}\right)\right)$ be another point of the intersection. Then at some point on the curve between $x_{1}$ and $x_{0}$, the curvature must be larger than the curvature of $\partial B$, while at some point on the curve between $x_{2}$ and $x_{1}$, the curvature must be smaller or equal to the curvature of $\partial B$. Again, this contradicts the monotonicity of the curvature in condition (iv). We conclude then that the curve $\{z=a(\rho), \rho \geq 1\}$ intersects $\partial B$ only at $x_{0}$.

The $z$-component of $x_{m}$ is given by $-\frac{1}{2} a\left(\rho_{0}\right)$. Let $G=B \cap\{z>0\} \subset\{0<$ $z<a(\rho), \rho \geq 2\}$ and define

$$
v(x)=w(x) \log 3\left(1+\left(a^{\prime}\left(\rho_{0}\right)\right)^{2}\right)^{1 / 2}-\log \left|x-x_{m}\right| \quad \text { on } G .
$$

Since $v$ is harmonic for $\frac{1}{2}\left(\partial^{2} / \partial \rho^{2}+\partial^{2} / \partial z^{2}\right)$, it obeys the maximum principle in $G$; therefore $v$ attains its maximum in $\bar{G}$ on $\partial G$. On $\partial G \cap\{z=0\}$, $v \leq-\log \frac{1}{2} a\left(\rho_{0}\right)$ since $w=0$ and $\log \left|x-x_{m}\right| \geq \log \frac{1}{2} a\left(\rho_{0}\right)$. It is easy to see that on $\partial G \cap \partial B, v$ attains its maximum at $x_{0}$ and

$$
v\left(x_{0}\right)=\log 3\left(1+\left(a^{\prime}\left(\rho_{0}\right)\right)^{2}\right)^{1 / 2}-\log \frac{3}{2} a\left(\rho_{0}\right)\left(1+\left(a^{\prime}\left(\rho_{0}\right)\right)^{2}\right)^{1 / 2}=-\log \frac{1}{2} a\left(\rho_{0}\right) .
$$

From these considerations, we conclude that $v$ attains its maximum in $\bar{G}$ at $x_{0}$. Thus $\partial v / \partial n\left(x_{0}\right) \geq 0$ or, equivalently,

$$
\left(\log 3\left(1+\left(a^{\prime}\left(\rho_{0}\right)\right)^{2}\right)^{1 / 2}\right) \frac{\partial w}{\partial n}\left(z_{0}\right) \geq \frac{1}{\frac{3}{2} a\left(\rho_{0}\right)\left(1+\left(a^{\prime}\left(\rho_{0}\right)\right)^{2}\right)^{1 / 2}} .
$$

Since $\sup _{\rho \geq 1} a^{\prime}(\rho)<\infty$, this proves the lower bound in (2.2).

Since

$$
w_{y}=\frac{1}{\left(1+\left(a^{\prime}\right)^{2}\right)^{1 / 2}} \frac{\partial w}{\partial n} \quad \text { and } \quad w_{\rho}=\frac{a^{\prime}}{\left(1+\left(a^{\prime}\right)^{2}\right)^{1 / 2}} \frac{\partial w}{\partial n},
$$

it follows from (2.2), symmetry considerations, and the fact that $\sup _{\rho \geq 1} a^{\prime}(\rho)<$ $\infty$ that there exist positive constants $\gamma_{1}$ and $\gamma_{2}$ such that

$$
\begin{array}{r}
\gamma_{1} / a(\rho) \leq w_{z} \leq \gamma_{2} / a(\rho) \\
\left|w_{\rho}\right| \leq \gamma_{2} / a(\rho)
\end{array} \quad \text { on }\{|z|=a(\rho) \text { and } \rho>1\}
$$

To prove the lemma, we must extend the estimates in (2.3) to the interior, $\{|z|<a(\rho)$ and $\rho>1\}$ (with new constants $c_{1}$ and $c_{2}$ in place of $\gamma_{1}$ and $\gamma_{2}$ ). Note that $w_{z}$ and $w_{\rho}$ are both harmonic for the operator $\frac{1}{2}\left(\partial^{2} / \partial \rho^{2}+\partial^{2} / \partial z^{2}\right)$. We will prove the extension of $(2.3)$ to the interior for $w_{z}$; the proof for $w_{\rho}$ is very similar to the proof that $w_{z} \leq c_{2} / a(\rho)$ in the interior.

Let $M=\{(\rho, z):|z|<a(\rho)$ and $\rho>1\}$ and let $u=w_{z}$. Then

$$
\frac{1}{2}\left(\partial^{2} / \partial \rho^{2}+\partial^{2} / \partial z^{2}\right) u=0
$$

in $M, u_{\rho}=0$ on $\partial M \cap\{\rho=1\}$, and, by (2.3),

$$
\gamma_{1} / a(\rho) \leq u(\rho, z) \leq \gamma_{2} / a(\rho) \text { on } \partial M-\{\rho=1\} .
$$


Let $(\rho(t), z(t))$ be a two-dimensional Brownian motion on $\{(\rho, z): \rho>1\}$ with reflection at $\{\rho=1\}$ and let $P_{\rho, z}$ and $E_{\rho, z}$ denote probabilities and expectations for the Brownian motion starting at $(\rho, z)$. Define

$$
\tau=\inf \{t \geq 0:|z(t)|=a(\rho(t))\} .
$$

Then

$$
u(\rho, z)=E_{\rho, z} u(\rho(\tau), z(\tau)) .
$$

We first prove the lower bound, that is, $u(\rho, z) \geq c_{1} / a(\rho)$. Let $A(\rho)=$ $\left\{\left(\rho^{\prime}, z\right) \in M: \rho / 2<\rho^{\prime}<3 / 2 \rho\right\}$ and let $\tau_{A(\rho)}=\inf \{t \geq 0:(\rho(t), z(t)) \notin$ $A(\rho)\}$. From condition (ii) on $a(\rho)$ in Theorem 2 , it follows that

$$
\sup _{\rho>1} \frac{a(k \rho)}{a(\rho)} \leq k, \quad \text { for } k \geq 1 \text {. }
$$

From (2.4)-(2.6) and the fact that $a$ is nondecreasing (condition (i) on $a(\rho)$ in Theorem 2), the lower bound in the lemma will follow if we prove that

$$
\inf _{|z|<a(\rho)} P_{\rho, z}\left(\tau_{A(\rho)}=\tau\right) \geq c>0, \quad \text { for large } \rho .
$$

Now fix $\rho_{0}$ and define

$$
B\left(\rho_{0}\right)=\left\{(\rho, z) \in R^{2}: \rho_{0} / 2<\rho<3 / 2 \rho_{0} \text { and }|z|<a\left(3 / 2 \rho_{0}\right)\right\} .
$$

Since $a$ is nondecreasing, $A\left(\rho_{0}\right) \subset B\left(\rho_{0}\right)$. Let $\tau_{B\left(\rho_{0}\right)}=\inf \{t \geq 0:(\rho(t), z(t))$ $\left.\notin B\left(\rho_{0}\right)\right\}$. Clearly,

$$
P_{\rho_{0}, z}\left(\tau_{A\left(\rho_{0}\right)}=\tau\right) \geq P_{\rho_{0}, z}\left(\left|z\left(\tau_{B\left(\rho_{0}\right)}\right)\right|=a\left(3 / 2 \rho_{0}\right)\right) .
$$

Define

$$
v(\rho, z)=\cos \left(\rho-\rho_{0}\right) \frac{\pi}{\rho_{0}} \frac{\exp \left(\pi z / \rho_{0}\right)+\exp \left(-\pi z / \rho_{0}\right)}{\exp \left(\pi a\left(3 / 2 \rho_{0}\right) / \rho_{0}\right)+\exp \left(-\pi a\left(3 / 2 \rho_{0}\right) / \rho_{0}\right)} .
$$

Then $\left(\partial^{2} / \partial \rho^{2}+\partial^{2} / \partial z^{2}\right) v=0$ and $0 \leq v \leq 1$ in $B\left(\rho_{0}\right)$. Also $v=0$ on $\rho=\rho_{0} / 2$ and on $\rho=3 / 2 \rho_{0}$. Therefore,

$$
\begin{aligned}
P_{\rho_{0}, z}\left(\left|z\left(\tau_{B\left(\rho_{0}\right)}\right)\right|\right. & \left.=a\left(3 / 2 \rho_{0}\right)\right) \geq v\left(\rho_{0}, z\right) \\
& =\frac{\exp \left(\pi z / \rho_{0}\right)+\exp \left(-\pi z / \rho_{0}\right)}{\exp \left(\pi a\left(3 / 2 \rho_{0}\right) / \rho_{0}\right)+\exp \left(-\pi a\left(3 / 2 \rho_{0}\right) / \rho_{0}\right)} .
\end{aligned}
$$

Now (2.7) follows from (2.8) and (2.9) and condition (ii) on $a(\rho)$ in Theorem 2.

We now turn to the upper bound. Introduce the polar coordinates $s=$ $\left(\rho^{2}+z^{2}\right)^{1 / 2}$ and $\phi=\tan ^{-1}(z / \rho)$ and let $(s(t), \phi(t))$ denote the Brownian motion $(\rho(t), z(t))$ in polar coordinates. By condition (ii) on $a(\rho)$ in Theorem 2 , it follows that there exists a $c>0$ such that $a(\rho) \leq c \rho$, for all $\rho>1$. Now fix $\rho_{0}$ and let $m>1$ be a real number to be fixed later on. Let $\phi_{0}=\tan ^{-1} c$ and $\phi_{1}=\tan ^{-1}(c+1)$. Define

$$
A\left(\rho_{0}\right)=\left\{(s, \phi): s>\rho_{0} / m,|\phi|<\phi_{1}\right\}
$$

and let $\tau_{A\left(\rho_{0}\right)}=\inf \left\{t \geq 0:(\rho(t), z(t)) \notin A\left(\rho_{0}\right)\right\}$. Define

$$
v(s, \phi)=\frac{\left(\rho_{0} / m\right)^{\pi / 2 \phi_{1}}}{\cos \left(\pi \phi_{0} / 2 \phi_{1}\right)} s^{-\pi / 2 \phi_{1}} \cos \frac{\pi \phi}{2 \phi_{1}} .
$$


Then

$$
\frac{1}{2}\left(\frac{\partial^{2}}{\partial \rho^{2}}+\frac{\partial^{2}}{\partial z^{2}}\right) v=\frac{1}{2}\left(\frac{\partial^{2}}{\partial s^{2}}+\frac{1}{s} \frac{\partial}{\partial s}+\frac{1}{s^{2}} \frac{\partial^{2}}{\partial \phi^{2}}\right) v=0
$$

and $v>0$ in $A\left(\rho_{0}\right)$. Also, $v=0$ on $\phi= \pm \phi_{1}, v\left(\rho_{0} / m, \phi\right) \geq 1$, for $|\phi|<\phi_{0}$, and $\lim _{s \rightarrow \infty} v(s, \phi)=0$. Therefore,

$$
\sup _{|z|<a\left(\rho_{0}\right)} P_{\rho_{0}, z}\left(s(\tau) \leq \frac{\rho_{0}}{m}\right) \leq \sup _{|\phi|<\phi_{0}} v\left(\rho_{0}, \phi\right) \leq c m^{-\pi / 2 \phi_{1}}
$$

Using the strong Markov property and iterating (2.10) gives

$$
\sup _{|z|<a\left(\rho_{0}\right)} P_{\rho_{0}, z}\left(s(\tau) \leq \frac{\rho_{0}}{m^{n}}\right) \leq\left(\frac{c}{m^{\pi / 2 \phi_{1}}}\right)^{n}, \quad n \geq 1 .
$$

Now if $(\rho, z) \in M$, then $|z| \leq c \rho$ and, thus, $s \leq \sqrt{1+c^{2}} \rho$. Using this and (2.11), we have

$$
\begin{aligned}
P_{\rho_{0}, z} & \left(\frac{\rho_{0}}{\sqrt{1+c^{2}} m^{n+1}} \leq \rho(\tau) \leq \frac{\rho_{0}}{\sqrt{1+c^{2}} m^{n}}\right) \\
& \leq P_{\rho_{0}, z}\left(\rho(\tau) \leq \frac{\rho_{0}}{\sqrt{1+c^{2}} m^{n}}\right) \leq P_{\rho_{0}, z}\left(s(\tau) \leq \frac{\rho_{0}}{m^{n}}\right) \leq\left(\frac{c}{m^{\pi / 2 \phi_{1}}}\right)^{n} .
\end{aligned}
$$

From (2.4), (2.5), (2.6), (2.12), and the fact that $a(\rho)$ is nondecreasing, we obtain

$$
\begin{aligned}
& u\left(\rho_{0}, z\right)=E_{\rho_{0}, z} u(\rho(\tau), z(\tau)) \\
& \quad \leq \frac{\gamma_{2}}{a\left(\rho\left(1+c^{2}\right)^{-1 / 2} m^{-1}\right)}+\sum_{n=1}^{\infty}\left(\frac{c}{m^{\pi / 2 \phi_{1}}}\right)^{n} \frac{\gamma_{2}}{a\left(\rho\left(1+c^{2}\right)^{-1 / 2} m^{-n-1}\right)} \\
& \quad=\frac{\gamma_{2}}{a(\rho)}\left[\frac{a(\rho)}{a\left(\rho\left(1+c^{2}\right)^{-1 / 2} m^{-1}\right)}+\sum_{n=1}^{\infty}\left(\frac{c}{m^{\pi / 2 \phi_{1}}}\right)^{n} \frac{a(\rho)}{a\left(\rho\left(1+c^{2}\right)^{-1 / 2} m^{-n-1}\right)}\right] \\
& \leq \frac{\gamma_{2}}{a(\rho)} \sqrt{1+c^{2}}\left[m+\sum_{n=1}^{\infty}\left(\frac{c}{m^{\pi / 2 \phi_{1}}}\right)^{n} m^{n+1}\right] .
\end{aligned}
$$

Since $\pi / 2 \phi_{1}>1$, by picking $m$ sufficiently large, the infinite series on the right-hand side of (2.13) converges. This gives the upper bound and completes the proof of the lemma.

Let $\bar{\rho}(r)$ solve

$$
\bar{\rho}^{\prime}=a(\bar{\rho}), \quad r>1, \quad \bar{\rho}(1)=1 .
$$

Let $\rho(r, w)$ denote the original variable $\rho$ as a function of the new variables $r$ and $w$.

Lemma 2. There exist constants $c_{1}, c_{2}>0$ such that

$$
c_{1} \bar{\rho}(r) \leq \rho(r, w) \leq c_{2} \bar{\rho}(r), \quad \text { for }(r, w) \in(1, \infty) \times(-1,1) .
$$

Proof. Since $d r=w_{z}(\rho, z) d \rho-w_{\rho}(\rho, z) d z$ and $d w=w_{z}(\rho, z) d z+$ $w_{\rho}(\rho, z) d \rho$, we obtain

$$
d \rho=\frac{w_{z}}{|\nabla w|^{2}}(\rho, z) d r+\frac{w_{\rho}}{|\nabla w|^{2}}(\rho, z) d w
$$

The lemma now follows easily from Lemma 1. 
The next lemma estimates the coefficients of $L$ in (2.1) in terms of the variable $r$.

Lemma 3. There exist constants $c_{1}, c_{2}>0$ such that the following inequalities hold for

$$
\begin{gathered}
\{(\rho, z): \rho>1 \text { and }|z|<a(\rho)\}=\{(r, w): r>1 \text { and }|w|<1\} \\
c_{1} \frac{a(\bar{\rho}(r))}{\bar{\rho}(r)} \leq \frac{w_{z}(\rho, z)}{\rho|\nabla w|^{2}(\rho, z)} \leq c_{2} \frac{a(\bar{\rho}(r))}{\bar{\rho}(r)} \\
\left|\frac{w_{\rho}(\rho, z)}{\rho|\nabla w|^{2}(\rho, z)}\right| \leq c_{2} \frac{a(\bar{\rho}(r))}{\bar{\rho}(r)} \\
c_{1}\left(\frac{a(\bar{\rho}(r))}{\bar{\rho}(r)}\right)^{2} \leq \frac{1}{\rho^{2}|\nabla w|^{2}(\rho, z)} \leq c_{2}\left(\frac{a(\bar{\rho}(r))}{\bar{\rho}(r)}\right)^{2}
\end{gathered}
$$

Proof. The lemma follows immediately from Lemmas 1 and 2 and (2.6).

For the rest of this paper, $P_{r, \theta, w}$ and $E_{r, \theta, w}$ will denote probabilities and expectations for the diffusion process $(r(t), \theta(t), w(t))$ corresponding to the operator $L$ in (2.1). Let $\sigma_{r}=\inf \{t \geq 0: r(t)=r\}$ and let $\tau=$ $\inf \{t \geq 0:|w(t)|=1\}$. Define $h_{0}(r, \theta, w)=P_{r, \theta, w}\left(\sigma_{1}<\tau\right)$. In the sequel, we will write $h_{0}(r, w)$ since $h_{0}$ does not depend on $\theta$. Define the $h_{0}$-transformed measure $P_{r, \theta, w}^{h_{0}}$ by

$$
\begin{aligned}
& P_{r, \theta, w}^{h_{0}}\left(\left\{(r(t), \theta(t), w(t)), 0 \leq t \leq \sigma_{1}\right\} \in \cdot\right) \\
& \quad=P_{r, \theta, w}\left(\left\{(r(t), \theta(t), w(t)), 0 \leq t \leq \sigma_{1}\right\} \in \cdot \mid \sigma_{1}<\tau\right) .
\end{aligned}
$$

The measure $P_{r, \theta, w}^{h_{0}}$ corresponds to the $h_{0}$-transformed operator $L^{h_{0}}$ defined by $L^{h_{0}} u=L\left(h_{0} u\right) / h_{0}$. Finally, let

$$
\nu_{r, \theta, w}^{h_{0}}\left(d \theta^{\prime}, d w^{\prime}\right)=P_{r, \theta, w}^{h_{0}}\left(\theta\left(\sigma_{1}\right) \in d \theta^{\prime}, w\left(\sigma_{1}\right) \in d w^{\prime}\right)
$$

denote the harmonic measure on $S^{d-1} \times(-1,1)$ for the conditioned process. Recall that $\Sigma=\left\{(r, \theta, w): r>1, \theta \in S^{d-1},|w|<1\right\}$. To prove the theorem, in terms of the coordinates $(r, \theta, w)$, what we must show is the following:

$$
\begin{aligned}
& \text { If } \int^{\infty} \frac{a(\rho)}{\rho^{2}} d \rho=\infty \text {, then } \mathrm{w}-\lim _{n \rightarrow \infty} \nu_{r_{n}, \theta_{n}, w_{n}}^{h_{0}} \text { exists for every } \\
& \text { sequence }\left\{\left(r_{n}, \theta_{n}, w_{n}\right)\right\}_{n=1}^{\infty} \subset \Sigma \text { which satisfies } \lim _{n \rightarrow \infty} r_{n}=\infty
\end{aligned}
$$

If $\int^{\infty} \frac{a(\rho)}{\rho^{2}} d \rho<\infty$, then $\mathrm{w}-\lim _{n \rightarrow \infty} \nu_{r_{n}, \theta_{n}, w_{n}}^{h_{0}}$ exists for a sequence $\left\{\left(r_{n}, \theta_{n}, w_{n}\right)\right\}_{n=1}^{\infty} \subset \Sigma$ which satisfies $\lim _{n \rightarrow \infty} r_{n}=\infty$ if and only if $\lim _{n \rightarrow \infty} \theta_{n}$ exists on $S^{d-1}$.

Before proving (2.15), we still need two more lemmas. 
Lemma 4. Let $\left\{\left(r_{n}, \theta_{n}, w_{n}\right)\right\}_{n=1}^{\infty} \subset \Sigma$ be a sequence satisfying $\lim _{n \rightarrow \infty} r_{n}=\infty$. Then the second marginal distribution,

$$
P_{r_{n}, \theta_{n}, w_{n}}^{h_{0}}\left(w\left(\sigma_{1}\right) \in d w\right)=\int_{S^{d-1}} \nu_{r_{n}, \theta_{n}, w_{n}}^{h_{0}}(d \theta, d w),
$$

of $\nu_{r_{n}, \theta_{n}, w_{n}}^{h_{0}}$ converges in the total variation norm as $n \rightarrow \infty$.

Proof. Let $\gamma_{r, \theta, w}^{(n)}\left(d w^{\prime}\right)=P_{r, \theta, w}^{h_{0}}\left(w\left(\sigma_{n}\right) \in d w^{\prime}\right)$, for $r>n, \theta \in S^{d-1}$, and $w \in(-1$,$) . Then, by using the coupling technique employed in [6], it is$ enough to show that there exist constants $c_{1}, c_{2}>0$ such that

$$
c_{1} \leq \frac{d \gamma_{n+1, \theta, w}^{(n)}}{d \gamma_{n+1, \theta^{\prime}, w^{\prime}}^{(n)}} \leq c_{2}, \quad \text { for all } n \geq 1, \theta, \theta^{\prime} \in S^{d-1} \text {, and } w, w^{\prime} \in(-1,1) \text {. }
$$

(See Theorem 4(ii) and its proof in [6].)

Let $f>0$ be a smooth function on $(-1,1)$ and let

$$
u_{n}(r, \theta, w)=\int_{-1}^{1} f\left(w^{\prime}\right) \gamma_{r, \theta, w}^{(n)}\left(d w^{\prime}\right),
$$

for $r>n, \theta \in S^{d-1}$, and $w \in(-1,1)$. Then $u_{n}$ is the minimal positive solution of $L^{h_{0}} u_{n}=0$ in $(n, \infty) \times S^{d-1} \times(-1,1)$ and $u_{n}(n, \theta, w)=f(w)$. Note that $u_{n}$ does not depend on $\theta$. Thus, we will write $u_{n}(r, w)$. Now (2.16) is equivalent to

$$
\begin{aligned}
& c_{1} \leq \frac{u_{n}(n+1, w)}{u_{n}\left(n+1, w^{\prime}\right)} \leq c_{2}, \text { where } c_{1}, c_{2}>0 \text { are independent of } \\
& n \geq 1, w, w^{\prime} \in(-1,1), \text { and } f .
\end{aligned}
$$

Let $v_{n}=u_{n} h_{0}$. Then $v_{n}$ is the minimal positive solution of $L v_{n}=0$ in $(n, \infty) \times S^{d-1} \times(1,1), v_{n}(n, \theta, w)=u_{n}(n, w) h_{0}(n, w)$, and $v_{n}(r, \theta, \pm 1)=$ 0 . Since $v_{n}$ does not depend on $\theta$, we will write $v_{n}(r, w)$. We have

$$
\frac{u_{n}(n+1, w)}{u_{n}\left(n+1, w^{\prime}\right)}=\frac{v_{n}(n+1, w)}{v_{n}\left(n+1, w^{\prime}\right)} \frac{h_{0}\left(n+1, w^{\prime}\right)}{h_{0}(n+1, w)} .
$$

The operator $L$, when restricted to the variables $r$ and $w$, has one half the Laplacian as its principal part and, by Lemma 3, has uniformly bounded firstorder terms. Since $v_{n}$ and $h_{0}$ depend only on $r$ and $w$, it follows from Harnack's inequality and the boundary Harnack principle [1] that there exist constants $c_{1}, c_{2}>0$ independent of $n \geq 1, w, w^{\prime} \in(-1,1), f$ such that

$$
c_{1} \frac{h_{0}(n+1, w)}{h_{0}\left(n+1, w^{\prime}\right)} \leq \frac{v_{n}(n+1, w)}{v_{n}\left(n+1, w^{\prime}\right)} \leq c_{2} \frac{h_{0}(n+1, w)}{h_{0}\left(n+1, w^{\prime}\right)} .
$$

This gives (2.17).

Lemma 5. Let $\left\{\left(r_{n}, \theta_{n}, w_{n}\right)\right\}_{n=1}^{\infty} \subset \Sigma$ be a sequence satisfying $\lim _{n \rightarrow \infty} r_{n}=\infty$.

(i) If $\int^{\infty} a(\rho) / \rho^{2} d \rho=\infty$, then $\int_{0}^{\sigma_{1}} a^{2}\left(\bar{\rho}(r(s)) / \bar{\rho}^{2}(r(s)) d s\right.$ under $P_{r_{n}}^{h_{0}}, \theta_{n}, w_{n}$ converges in distribution as $n \rightarrow \infty$ to the atom at infinity.

(ii) If $\int^{\infty} a(\rho) / \rho^{2} d \rho<\infty$, then $\int_{0}^{\sigma_{1}} a^{2}\left(\bar{\rho}(r(s)) / \bar{\rho}^{2}(r(s)) d s\right.$ under $P_{r_{n}, \theta_{n}, w_{n}}^{h_{0}}$ is tight on $[0, \infty)$ as $n \rightarrow \infty$. 
Proof. By a change of variables, $\int_{1}^{\infty} a(\rho) / \rho^{2} d \rho=\int_{0}^{\infty} a^{2}(\bar{\rho}(s)) / \bar{\rho}^{2}(s) d s$. By condition (ii) on $a(\rho)$ in Theorem 2 , it follows that $a^{2}(\bar{\rho}(s)) / \bar{\rho}^{2}(s)$ is monotone nonincreasing in $s$. Thus, we can restate the integral condition as a condition on an infinite series:

(2.18) If the condition in part (i) holds, then $\sum_{n=1}^{\infty} \frac{a^{2}(\bar{\rho}(n))}{\bar{\rho}^{2}(n)}=\infty$.

(2.19) If the condition in part (ii) holds, then $\sum_{n=1}^{\infty} \frac{a^{2}(\bar{\rho}(n))}{\bar{\rho}^{2}(n)}<\infty$.

Part (i). To prove part (i), we will show that

$$
\limsup _{r \rightarrow \infty} E_{r, w}^{h_{0}} \exp \left(-\int_{0}^{\sigma_{1}} \frac{a^{2}(\bar{\rho}(r(s)))}{\bar{\rho}^{2}(r(s))} d s\right)=0 .
$$

Let $\gamma_{n}=\sigma_{n-1} \wedge \sigma_{n+1} \wedge \tau$ and let $A_{n}=a^{2}(\bar{\rho}(n)) / \bar{\rho}^{2}(n)$. Then, clearly

$$
E_{n, w}^{h_{0}} \exp \left(-\int_{0}^{\sigma_{1}} \frac{a^{2}(\bar{\rho}(r(s)))}{\bar{\rho}^{2}(r(s))} d s\right) \leq \prod_{k=2}^{n} \sup _{w \in(-1,1)} E_{k, w}^{h_{0}} e^{-A_{k+1} \gamma_{k}} .
$$

Since

$$
\left(\frac{\partial}{\partial t}+L\right)\left(e^{-A_{n} t} h_{0}(r, w)\right)=-A_{n} e^{-A_{n} t} h_{0}(r, w),
$$

we have

$$
\begin{aligned}
E_{n, w}^{h_{0}} e^{-A_{n+1} \gamma_{n}} & =\frac{1}{h_{0}(n, w)} E_{n, w} e^{-A_{n+1} \gamma_{n}} h_{0}\left(r\left(\gamma_{n}\right), w\left(\gamma_{n}\right)\right) \\
& =1-A_{n+1} \frac{1}{h_{0}(n, w)} E_{n, w} \int_{0}^{\gamma_{n}} h_{0}(r(s), w(s)) e^{-A_{n+1} s} d s .
\end{aligned}
$$

We may assume without loss of generality that

$$
\lim _{n \rightarrow \infty} A_{n}=0 \text {. }
$$

By (2.18), $\sum_{n=1}^{\infty} A_{n}=\infty$. Therefore, (2.20) will follow from (2.21) and (2.22) if we show that

$$
\liminf _{n \rightarrow \infty} \frac{1}{h_{0}(n, w)} E_{n, w} \int_{0}^{\gamma_{n}} h_{0}(r(s), w(s)) e^{-A_{n+1} s} d s>0 .
$$

We have

$$
\begin{aligned}
& \frac{1}{h_{0}(n, w)} E_{n, w} \int_{0}^{\gamma_{n}} h_{0}(r(s), w(s)) e^{-A_{n+1} s} d s \\
& \quad \geq \frac{1}{h_{0}(n, w)} E_{n, w} \int_{0}^{\gamma_{n} \wedge 1 / A_{n+1}} h_{0}(r(s), w(s)) e^{-A_{n+1} s} d s \\
& \quad \geq \frac{e^{-1}}{h_{0}(n, w)} E_{n, w} \int_{0}^{\gamma_{n} \wedge 1 / A_{n+1}} h_{0}(r(s), w(s)) d s \\
& \quad \geq \frac{e^{-1}}{h_{0}(n, w)} E_{n, w} \int_{0}^{\gamma_{n}} h_{0}(r(s), w(s)) d s \\
& \quad-e^{-1}\left(\sup _{\substack{n-1<r<n+1 \\
-1<w^{\prime}<1}} h_{0}\left(r, w^{\prime}\right)\right) \frac{1}{h_{0}(n, w)} E_{n, w} \gamma_{n} 1_{\left\{\gamma_{n}>1 / A_{n+1}\right\}} .
\end{aligned}
$$


We will show that the first term on the right-hand side of $(2.25)$ is bounded below by a positive constant and that the second term converges to zero as $r \rightarrow \infty$, uniformly in $w$.

Let $B_{\varepsilon}(r, w)$ denote the ball of radius $\varepsilon$ centered at $(r, w)$. For $\varepsilon>0$, there exists a constant $c>0$ independent of $r$ and $w$ such that

$$
\begin{aligned}
P_{r, w}\left((r(t), w(t)) \in B_{\varepsilon}(r, w) \text { for } 0 \leq t \leq 1\right) \geq c, \\
\\
\text { if } B_{\varepsilon}(r, w) \subset(1, \infty) \times(-1,1) .
\end{aligned}
$$

This can be proved easily by applying the Cameron-Martin transformation to Brownian motion and using the Schwarz inequality, the fact that the drift coefficients in $L$ are uniformly bounded, and the fact that the result is true for Brownian motion. From (2.26) and Harnack's inequality, it follows that for $w$ away from \pm 1 and $n \geq 1$, the first term on the right-hand side of (2.25) is bounded below by a positive constant. We must extend this to $w$ near \pm 1 .

For the rest of the proof of this lemma, whenever we write $L$, what we actually mean is the restriction of $L$ to functions depending only on $r$ and $w$. We will only work with the Markov process $(r(t), w(t))$; thus we will write $P_{r, w}^{h_{0}}$ and $E_{r, w}^{h_{0}}$ for $P_{r, \theta, w}^{h_{0}}$ and $E_{r, \theta, w}^{h_{0}}$. Let $b_{1}(r, w)$ and $b_{2}(r, w)$ denote the coefficients of $\partial / \partial r$ and $\partial / \partial w$, respectively, in the operator $L$. It is easy to show that $w_{\rho}(\rho, z) \leq 0$ for $0<z<a(\rho)$ and $w_{\rho}(\rho, z) \geq 0$ for $-a(\rho)<z<0$. (Apply the maximum principle to the harmonic function $w_{\rho}$ and use symmetry.) Similarly, $w_{z}(\rho, z) \geq 0$ for $-1<w<1$. Therefore, $b_{2}(r, w) \leq 0$ for $0<w<1$ and $b_{2}(r, w) \geq 0$ for $-1<w<0$, and $b_{1}(r, w) \geq 0$ for $-1<w<1$. Let $c_{1}=\sup _{r \geq 1,-1<w<1} b_{1}(r, w)$. Define the operator

$$
\mathscr{A}_{n}=\frac{1}{2}\left(\frac{\partial^{2}}{\partial r^{2}}+\frac{\partial^{2}}{\partial w^{2}}\right)+c_{1} \operatorname{sgn}(r-n) \frac{\partial}{\partial r}
$$

and let $P_{r, w}^{\mathscr{A}_{n}}$ denote probabilities for the diffusion corresponding to $\mathscr{A}_{n}$. Let

$$
u_{n}(r, w)=P_{r, w}^{\mathscr{A}_{n}}\left(w(t)=0 \text { for some } t<\sigma_{n-1 / 2} \wedge \sigma_{n+1 / 2} \wedge \tau\right) .
$$

Then $\mathscr{A}_{n} u_{n}=0$ in $\left(n-\frac{1}{2}, n+\frac{1}{2}\right) \times(0,1), u_{n}\left(n \pm \frac{1}{2}, w\right)=0, u_{n}(r, 1)=0$, and $u_{n}(r, 0)=1$. Note that, in fact, $u_{n}$ is independent of $n$. Thus, by the Hopf maximum principle, there exists a $c>0$ such that $u_{n}(n, w) \geq c(1-w)$ for $0<w<1$ and $n \geq 1$. Let

$$
v_{n}(r, w)=P_{r, w}\left(w(t)=0 \text { for some } t<\sigma_{n-1 / 2} \wedge \sigma_{n+1 / 2} \wedge \tau\right) .
$$

Then $L v_{n}=0$ in $\left(n-\frac{1}{2}, n+\frac{1}{2}\right) \times(0,1), v_{n}\left(n \pm \frac{1}{2}, w\right)=0, v_{n}(r, 1)=0$, and $v_{n}(r, 0)=1$. We have

$$
L\left(v_{n}-u_{n}\right)=\left(c_{1} \operatorname{sgn}(r-n)-b_{1}(r, w)\right)\left(u_{n}\right)_{r}-b_{2}(r, w)\left(u_{n}\right)_{w} .
$$

Using symmetry, it is easy to show that $\left(u_{n}\right)_{r}(r, w) \leq 0$ for $n<r<n+\frac{1}{2}$ and $\left(u_{n}\right)_{r}(r, w) \geq 0$ for $n-\frac{1}{2}<r<n$. (We should point out that the discontinuity at $r=n$ causes no problem. Indeed, the present situation is equivalent to the situation where the diffusion is restricted to $\left(n, n+\frac{1}{2}\right) \times(-1,1)$ with normal reflection at $r=n$.) Similarly, $\left(u_{n}\right)_{w}(r, w) \leq 0$ for $0<w<1$. Thus $L\left(v_{n}-u_{n}\right) \leq 0$ in $\left(n-\frac{1}{2}, n+\frac{1}{2}\right) \times(0,1)$ and, by the maximum principle, 
$v_{n} \geq u_{n}$. Thus, $v_{n}(n, w) \geq u_{n}(n, w) \geq c(1-w)$, for $0 \leq w \leq 1$. By symmetry then, we conclude that there exists a $c>0$ such that

$$
\begin{array}{r}
P_{n, w}\left(w(t)=0 \text { for some } t<\sigma_{n-1 / 2} \wedge \sigma_{n+1 / 2} \wedge \tau\right) \geq c\left(1-w^{2}\right), \\
\text { for } n \geq 2 \text { and } w \in(-1,1) .
\end{array}
$$

Now define

$$
\widehat{\mathscr{A}_{n}}=\frac{1}{2}\left(\frac{\partial^{2}}{\partial r^{2}}+\frac{\partial^{2}}{\partial w^{2}}\right)+c_{1} \operatorname{sgn}(r-n) \frac{\partial}{\partial r}-c_{2} \operatorname{sgn}(w) \frac{\partial}{\partial w},
$$

where $c_{2}=-\inf _{r>1,0<w<1} b_{2}(r, w)>0$, and let $P_{r, w}^{\mathscr{x}_{n}}$ denote probabilities for the diffusion corresponding to $\widehat{\mathscr{A}_{n}}$. Let

and let

$$
\hat{u}_{n}(r, w)=P_{r, w}^{\mathscr{\vartheta}_{n}}\left(\sigma_{n-1 / 2} \wedge \sigma_{n+1 / 2}<\tau\right)
$$

$$
\hat{v}_{n}(r, w)=P_{r, w}\left(\sigma_{n-1 / 2} \wedge \sigma_{n+1 / 2}<\tau\right) .
$$

Then an argument very similar to the one above shows that there exists a $c>0$ such that

$$
P_{n, w}\left(\sigma_{n-1 / 2} \wedge \sigma_{n+1 / 2}<\tau\right) \leq c\left(1-w^{2}\right), \quad \text { for } n \geq 2 \text { and } w \in(-1,1) .
$$

From (2.28), Harnack's inequality and the boundary Harnack principle, it follows that there exists a $c>0$ such that

$$
h_{0}(n, w) \leq c\left(1-w^{2}\right) h_{0}(n, 0), \quad \text { for } n \geq 2 \text { and } w \in(-1,1) .
$$

Using the strong Markov property with (2.26), (2.27), and (2.29), it follows now that the first term on the right-hand side of $(2.25)$ is bounded below by a positive constant, independent of $n$ and $w$.

We now show that the second term on the right-hand side of $(2.25)$ converges to zero as $n \rightarrow \infty$. Let $c_{2}=-\inf _{r \geq 1,0<w<1} b_{2}(r, w)>0$ as above and define the operator $\mathscr{A}=(1 / 2) d^{2} / d w^{2}-c_{2} \operatorname{sgn}(w) d / d w$. Let $P_{w}^{\mathscr{A}}$ denote probabilities for the diffusion corresponding to $\mathscr{A}$. Then by the IkedaWatanabe comparison theorem [4] and symmetry considerations, it follows that $P_{w}^{\mathscr{A}}(\tau>t) \geq P_{r, w}\left(\gamma_{n}>t\right)$. Let $\lambda_{0}>0$ and $\psi(w)$ denote the leading eigenvalue and the corresponding positive eigenfunction for $-\mathscr{A}$ in $(-1,1)$ with the Dirichlet boundary condition. By the parabolic maximum principle, $P_{w}^{\mathscr{A}}(\tau>t) \leq c \psi(w) e^{-\lambda_{0} t}$, for $t \geq 1$ and some constant $c>0$. Thus

$$
\begin{aligned}
E_{n, w} \gamma_{n} 1_{\left\{y_{n}>1 / A_{n+1}\right\}} & \leq \sum_{n>1 / A_{n+1}-1}(n+1) P_{n, w}\left(\gamma_{n}>n\right) \\
& \leq c \psi(w) \sum_{n>1 / A_{n+1}-1}(n+1) e^{-n \lambda_{0}} \equiv c_{n} \psi(w),
\end{aligned}
$$

where, by (2.23), $\lim _{n \rightarrow \infty} c_{n}=0$. Thus, for the second term on the right-hand side of (2.25), we have the estimate

$$
\sup _{\substack{n-1<r<n+1 \\-1<w^{\prime}<1}} h_{0}\left(r, w^{\prime}\right) \frac{1}{h_{0}(n, w)} E_{n, w} \gamma_{n} 1_{\left\{\gamma_{n}>1 / A_{n+1}\right\}} \leq \frac{c_{n} \psi(w)}{f_{n}(n, w)}
$$

where

$$
f_{n}(r, w)=\frac{h_{0}(r, w)}{\sup _{n-1<r^{\prime}<n+1,-1<w^{\prime}<1} h_{0}\left(r^{\prime}, w^{\prime}\right)} .
$$


We will show that there exists a constant $c>0$ such that

$$
f_{n}(n, w) \geq c\left(1-w^{2}\right), \quad \text { for all } n \text { and } w \in(-1,1) .
$$

Since $\psi$ is differentiable and vanishes at $w= \pm 1$, it will follow from (2.31) that the left-hand side of (2.30) converges to zero as $n \rightarrow \infty$, uniformly in $w$. This will complete the proof of part (i).

From Harnack's inequality, it is clear that (2.31) holds for $w$ away from \pm 1 . We must extend this to $w$ near \pm 1 . Let $g_{n}$ be the minimal positive solution of $L g_{n}=0$ in $(n-1, \infty) \times(-1,1), g_{n}(r, \pm 1)=0$, and $g_{n}(n-1, w)=$ $\delta 1_{(-1 / 2,1 / 2)}(w)$, where $\delta>0$. By Harnack's inequality and the maximum principle, if $\delta$ is chosen sufficiently small, then

$$
f_{n}(n, w) \geq g_{n}(n, w), \quad \text { for all } n \text { and } w \in(-1,1) .
$$

Let $G_{n}$ be the minimal positive solution of $L G_{n}=0$ in $(n-1, \infty) \times(-1,1)$, $G_{n}(r, \pm 1)=0$, and $G_{n}(n-1, w)=\delta$. Below we will show that there exists a $c>0$ such that

$$
g_{n}(n, 0) \geq c \text { for all } n .
$$

From this it follows that $g_{n}(n, 0) / G_{n}$ is bounded away from zero and thus, by Harnack's inequality and the boundary Harnack principle, there exists a $c>0$ such that

$$
g_{n}(n, w) \geq c G_{n}(n, w) \text { for all } n \text { and } w \in(-1,1) .
$$

Let $H_{n}$ be the minimal positive solution of

$$
\left(\frac{1}{2} \frac{\partial^{2}}{\partial r^{2}}+\frac{1}{2} \frac{\partial^{2}}{\partial w^{2}}+c_{1} \frac{\partial}{\partial r}\right) H_{n}=0
$$

in $(n-1, \infty) \times(-1,1), H_{n}(r, \pm 1)=0, H_{n}(n-1, w)=\delta$, where, as before, $c_{1}=\sup _{r \geq 1,-1<w<1} b_{1}(r, w)$ and $b_{1}$ is the coefficient of $\partial / \partial r$ in $L$. Then an argument similar to the one above for $u_{n}$ and $v_{n}$ shows that $L\left(G_{n}-H_{n}\right) \leq 0$. Therefore, by the maximum principle,

$$
G_{n} \geq H_{n} .
$$

However, note that $H_{n}(n, w)$ is independent of $n$. Therefore, by the Hopf maximum principle, there exists a $c>0$ such that

$$
H_{n}(n, w) \geq c\left(1-w^{2}\right) \text { for all } n \text { and } w \in(-1,1) .
$$

Now (2.31) follows from (2.32) and (2.34)-(2.36).

To complete the proof of part (i) we must show (2.33). Let $\hat{g}_{n}$ denote the solution to

$$
\left(\frac{1}{2} \frac{\partial^{2}}{\partial r^{2}}+\frac{1}{2} \frac{\partial^{2}}{\partial w^{2}}+c_{1} \frac{\partial}{\partial r}\right) \hat{g}_{n}=0
$$

in $(n-1, \infty) \times\left(-\frac{1}{2}, \frac{1}{2}\right), \hat{g}_{n}\left(r, \pm \frac{1}{2}\right)=0$, and $\hat{g}_{n}(n-1, w)=\delta$. An argument similar to several used above shows that $L\left(g_{n}-\hat{g}_{n}\right) \leq 0$ and thus, by the maximum principle, $g_{n} \geq \hat{g}_{n}$ in $(n-1, \infty) \times\left(-\frac{1}{2}, \frac{1}{2}\right)$. But $\hat{g}_{n}(n, 0)>0$ and is independent of $n$. This gives (2.33).

Part (ii). We will show that there exists a constant $c>0$ such that

$$
E_{n+1, w}^{h_{0}} \int_{0}^{\sigma_{1}} 1_{(n, n+1)}(r(s)) d s \leq c, \quad \text { for all } n \text { and } w \in(-1,1) .
$$


From this, it will follow that

$$
E_{r, w}^{h_{0}} \int_{0}^{\sigma_{1}} \frac{a^{2}(\bar{\rho}(r(s)))}{\bar{\rho}^{2}(r(s))} d s \leq c \sum_{n=1}^{\infty} \frac{a^{2}(\bar{\rho}(n))}{\bar{\rho}^{2}(n)}, \quad \text { for } r \geq 1 \text { and } w \in(-1,1) .
$$

This proves tightness since, by (2.19), the series on the right-hand side of (2.38) is convergent. It remains to prove (2.37).

Let

$$
u_{n}(r, w)=E_{r, w}^{h_{0}} \int_{0}^{\sigma_{1}} 1_{(n, n+1)}(r(s)) d s .
$$

Then $u_{n}$ is the minimal positive solution of $L^{h_{0}} u_{n}=-1_{(n, n+1)}(r)$ in $(1, \infty) \times$ $(-1,1)$ and $u_{n}(1, w)=0$. Let $v_{n}=u_{n} h_{0}$. Then $v_{n}$ is the minimal positive solution of $L v_{n}=-h_{0} \cdot 1_{(n, n+1)}(r)$ in $(1, \infty) \times(-1,1), v_{n}(1, w)=0$, and $v_{n}(r, \pm 1)=0$. Therefore, to prove (2.37), we must show that

$$
v_{n}(n+1, w) / h_{0}(n+1, w) \leq c .
$$

Let $G\left(r, w, r^{\prime}, w^{\prime}\right)$ denote the Green's function for $L$ in $(1, \infty) \times(-1,1)$ with the Dirichlet boundary condition at $w= \pm 1$ and $r=1$. Then

$$
v_{n}(n+1, w)=\int_{-1}^{1} d w^{\prime} \int_{1}^{\infty} d r^{\prime} G\left(n+1, w, r^{\prime}, w^{\prime}\right) h\left(r^{\prime}, w^{\prime}\right) 1_{(n, n+1)}\left(r^{\prime}\right) .
$$

Now

$$
\int_{-1}^{1} d w^{\prime} \int_{1}^{\infty} d r^{\prime} G\left(n+1, w, r^{\prime}, w^{\prime}\right)=E_{n+1, w} \tau \wedge \sigma_{1} .
$$

Therefore,

$$
v_{n}(n+1, w) / h_{0}(n+1, w) \leq E_{n+1, w} \tau \wedge \sigma_{1} / F_{n}(n+1, w),
$$

where

$$
F_{n}(r, w)=\frac{h_{0}(r, w)}{\sup _{n<r^{\prime}<n+1,-1<w^{\prime}<1} h_{0}\left(r^{\prime}, w^{\prime}\right)} .
$$

Now $F_{n}$ is almost identical to $f_{n}$ in part (i) and, analogous to (2.31), there exists a $c>0$ such that

$$
F_{n}(n+1, w) \geq c\left(1-w^{2}\right), \quad \text { for all } n \text { and } w \in(-1,1) .
$$

Recall from part (i) that

$$
\mathscr{A}=\frac{1}{2} \frac{d^{2}}{d w^{2}}-c_{2} \operatorname{sgn}(w) \frac{d}{d w}, \quad \text { where } c_{2}=\inf _{\substack{r \geq 1 \\ 0<w<1}} b_{2}(r, w) .
$$

By the Ikeda-Watanabe comparison theorem and symmetry considerations, it follows that $E_{n+1, w} \tau \wedge \sigma_{1} \leq E_{w}^{\mathscr{A}} \tau$. Since $E_{w}^{\mathscr{A}} \tau \leq c\left(1-w^{2}\right)$, for some $c>0$, we obtain

$$
E_{n+1, w} \tau \wedge \sigma_{1} \leq c\left(1-w^{2}\right), \quad \text { for all } n \text { and } w \in(-1,1) .
$$

Now (2.39) follows from (2.40)-(2.42). This completes the proof of Lemma 5.

We are now in a position to complete the proof of Theorem 2.

Proof of Theorem 2. To prove the theorem, we need to prove (2.15). We construct the skew product representation for the diffusion corresponding to $L^{h_{0}}=$ 
$L+\left(\nabla h_{0} / h_{0}\right) \cdot \nabla$ as follows. For each $(r, \theta, w) \in \Sigma$, consider a probability space with measure $P_{r, \theta, w}^{h_{0}}$ on which live stochastic processes $(r(t), w(t))$ and $\phi(t)$ and stopping times $\sigma_{1}=\inf \{t \geq 0: r(t)=1\}$ and $\tau=\inf \{t \geq 0:|w(t)|=1\}$ such that $(r(t), w(t))$ is independent of $\phi(t),(r(t), w(t))$ is a Markov process up to time $\sigma_{1} \wedge \tau$ starting from $(r, w)$ and corresponding to the operator obtained from $L^{h_{0}}$ by deleting terms involving differentiation in $\theta$, and $\phi(t)$ is a Brownian motion on $S^{d-1}$ starting from $\theta$. Let

$$
A(r, w)=2 / \rho^{2}|\nabla w|^{2}(\rho, z)
$$

and define

$$
\gamma(t)=\int_{0}^{t} A(r(s), w(s)) d s .
$$

Finally, iet $\theta(t)=\phi(\gamma(t))$. Then $(r(t), \theta(t), w(t))$ under $P_{r, \theta, w}^{h_{0}}$ is the skewproduct representation for the diffusion on $\Sigma$ corresponding to $L^{h_{0}}$. We can now write the harmonic measure as

$$
\begin{aligned}
\nu_{r, \theta, w}^{h_{0}}\left(d \theta^{\prime}, d w^{\prime}\right) & =P_{r, \theta, w}^{h_{0}}\left(\theta\left(\sigma_{1}\right) \in d \theta^{\prime}, w\left(\sigma_{1}\right) \in d w^{\prime}\right) \\
& =P_{r, \theta, w}^{h_{0}}\left(\phi\left(\gamma\left(\sigma_{1}\right)\right) \in d \theta^{\prime}, w\left(\sigma_{1}\right) \in d w^{\prime}\right) .
\end{aligned}
$$

For the proof of (2.15), it will be convenient to define for each $\theta \in S^{d-1}$, a Brownian motion $\phi_{\theta}(t)$ on $S^{d-1}$ starting at $\theta$ and living on a probability space with a measure $P$. The corresponding expectation will be denoted by $E$. We first prove (2.15a). Let $f$ be a bounded continuous function on $S^{d-1} \times$ $[-1,1]$ and let $\left\{\left(r_{n}, \theta_{n}, w_{n}\right)\right\}_{n=1}^{\infty} \subset \Sigma$ satisfy $\lim _{n \rightarrow \infty} r_{n}=\infty$. Define the probability measure $m_{n}(d t, d w)=P_{r_{n}, \theta_{n}, w_{n}}^{h_{0}}\left(\gamma\left(\sigma_{1}\right) \in d t, w\left(\sigma_{1}\right) \in d w\right)$. Since $\phi$ is independent of $\gamma\left(\sigma_{1}\right)$ and $w\left(\sigma_{1}\right)$, we can write

$$
\int f(\theta, w) \nu_{r_{n}, \theta_{n}, w_{n}}^{h_{0}}(d \theta, d w)=\int F_{\theta_{n}}(t, w) m_{n}(d t, d w),
$$

where $F_{\theta_{n}}(t, q)=E f\left(\phi_{\theta_{n}}(t), w\right)$. Since the distribution of the Brownian motion $\phi_{\theta}(t)$ on $S^{d-1}$ converges in total variation norm to normalized Lebesgue measure, uniformly in $\theta$, it follows that

$$
F(w) \equiv \lim _{t \rightarrow \infty} F_{\theta}(t, w) \text { exists uniformly in } \theta \text { and } w .
$$

By Lemma 3(iii), Lemma 5, and (2.43), it follows that the first marginal of $m_{n}$ converges weakly to the atom at infinity as $n \rightarrow \infty$ and, by Lemma 4 , the second marginal of $m_{n}$ converges. These facts in conjunction with (2.45) show that the right-hand side of (2.44) converges as $n \rightarrow \infty$. Thus, the left-hand side of (2.44) converges for all continuous bounded $f$, which proves (2.15a).

We now turn to $(2.15 \mathrm{~b})$. It suffices to prove the following two statements:

$$
\begin{aligned}
& \text { If }\left\{\left(r_{n}, \theta_{n}, w_{n}\right)\right\}_{n=1}^{\infty} \subset \sum \text { satisfies } \lim _{n \rightarrow \infty} r_{n}=\infty \text { and } \\
& \lim _{n \rightarrow \infty} \theta_{n} \text { exists, then w- } \lim _{n \rightarrow \infty} \nu_{r_{n}, \theta_{n}, w_{n}}^{h_{0}} \text { exists. }
\end{aligned}
$$

If $\left\{\left(r_{n}, \theta_{n}, w_{n}\right)\right\}_{n=1}^{\infty}$ and $\left\{\left(r_{n}^{\prime}, \theta_{n}^{\prime}, w_{n}^{\prime}\right)\right\}_{n=1}^{\infty}$ are sequences in $\Sigma$ satisfying

$$
\lim _{n \rightarrow \infty} r_{n}=\lim _{n \rightarrow \infty} r_{n}^{\prime}=\infty, \bar{\theta}_{1} \equiv \lim _{n \rightarrow \infty} \theta_{n} \text { and } \bar{\theta}_{2} \equiv \lim _{n \rightarrow \infty} \theta_{n}^{\prime} \text { exist }
$$


and $\bar{\theta}_{1} \neq \bar{\theta}_{2}$, then

$$
\bar{\nu}_{1} \equiv \mathrm{w}-\lim _{n \rightarrow \infty} \nu_{r_{n}, \theta_{n}, w_{n}}^{h_{0}} \neq \bar{\nu}_{2} \equiv \mathrm{w}-\lim _{n \rightarrow \infty} \nu_{r_{n}^{\prime}, \theta_{n}^{\prime}, w_{n}^{\prime}}^{h_{0}} .
$$

We begin with (2.47). From Lemma 3(iii), Lemma 5, (2.41), and the skewproduct representation, the first marginal of $\bar{\nu}_{i}$ must be of the form $\int_{0}^{\infty} P\left(\phi_{\bar{\theta}_{i}}(t) \in \cdot\right) \mu_{i}(d t)$, where $\mu_{i}(d t)$ is a probability measure on $[0, \infty)$. However, the density of $\int_{0}^{\infty} P\left(\phi_{\bar{\theta}_{i}}(t) \in \cdot\right) \mu_{i}(d t)$ attains its unique maximum at $\bar{\theta}_{i}$, and $\bar{\theta}_{1} \neq \bar{\theta}_{2}$; thus $\bar{\nu}_{1} \neq \bar{\nu}_{2}$.

We now turn to $(2.46)$. The same proof used to prove (2.38) shows that, analogous to $(2.38)$,

$$
\begin{aligned}
& E_{r, \theta, w}^{h_{0}} \int_{0}^{\sigma_{m}} \frac{a^{2}(\bar{\rho}(r(s)))}{\bar{\rho}^{2}(r(s))} d s \leq c \sum_{n=m}^{\infty} \frac{a^{2}(\bar{\rho}(n))}{\bar{\rho}^{2}(n)} \\
& \qquad \text { for } r \geq m, \theta \in S^{d-1}, \text { and } w \in(-1,1) .
\end{aligned}
$$

Let $\|\cdot\|$ denote a metric on the space of probability measure on $S^{d-1}$ which is compatible with the topology of weak convergence and let $\|\cdot\|_{\text {var }}$ denote the total variation norm on the space of measures on $[-1,1]$. Since the series on the right-hand side of (2.48) is a convergent one, it follows from Lemma 3(iii), (2.43), and (2.48) that for each $\varepsilon>0$, there exists an $m_{\varepsilon}$ such that for $m \geq m_{\varepsilon}$

$$
\begin{aligned}
& \left\|P_{r, \theta, w}^{h_{0}}\left(\phi\left(\gamma\left(\sigma_{m}\right)\right) \in \cdot\right)-\delta_{(\theta)}(\cdot)\right\|<\varepsilon, \\
& \qquad \text { for } r \geq m, \theta \in S^{d-1}, \text { and } w \in(-1,1) .
\end{aligned}
$$

The same proof as in Lemma 4 shows that there exists a probability measure $\eta$ on $(-1,1)$ such that for any $m \geq 1$,

$$
\lim _{r \rightarrow \infty}\left\|P_{r, \theta, w}^{h_{0}}\left(w\left(\sigma_{m}\right) \in \cdot\right)-\eta\right\|_{\mathrm{var}}=0
$$

$$
\text { uniformly over } \theta \in S^{d-1} \text { and } w \in(-1,1) \text {. }
$$

Applying the strong Markov property, we have for any $m>1$,

$$
\begin{aligned}
& \nu_{r_{n}, \theta_{n}, w_{n}}(d \theta, d w) \\
& \quad=\int \nu_{m, \theta^{\prime}, w^{\prime}}(d \theta, d w) P_{r_{n}, \theta_{n}, w}^{h_{0}}\left(\phi\left(\gamma\left(\sigma_{m}\right)\right) \in d \theta^{\prime}, w\left(\sigma_{m}\right) \in d w^{\prime}\right) .
\end{aligned}
$$

Thus, if $f$ is a continuous function on $S^{d-1} \times[-1,1]$, then

$$
\begin{aligned}
& \int f(\theta, w) \nu_{r_{n}, \theta_{n}, w_{n}}(d \theta, d w) \\
& \quad=\int F_{m}\left(\theta^{\prime}, w^{\prime}\right) P_{r_{n}, \theta_{n}, w_{n}}^{h_{n_{0}}}\left(\phi\left(\gamma\left(\sigma_{m}\right)\right) \in d \theta^{\prime}, w\left(\sigma_{m}\right) \in d w^{\prime}\right),
\end{aligned}
$$

where $F_{m}\left(\theta^{\prime}, w^{\prime}\right)=\int f(\theta, w) \nu_{m, \theta^{\prime}, w^{\prime}}(d \theta, d w)$. By the translation invariance of Brownian motion,

$$
F_{m}\left(\theta^{\prime}+\delta, w^{\prime}\right)=\int f(\theta+\delta, w) \nu_{m, \theta^{\prime}, w^{\prime}}(d \theta, d w) .
$$

Therefore, the family $\left\{F_{m}(\cdot, w), m>1, w \in(-1,1)\right\}$ of functions on $S^{d-1}$ is equicontinuous. Now an easy argument using this fact and (2.49) and (2.50) shows that the right-hand side and, thus, also the left-hand side, of $(2.51)$ converges as $n \rightarrow \infty$. This proves (2.46). 


\section{REFERENCES}

1. A. Ancona, Principe de Harnack à la frontiére et théorème de Fatou pour un opérateur elliptique dans un domaine Lipschitzien, Ann. Inst. Fourier (Grenoble) 28 (1978), 169-213.

2. J. L. Doob, Conditioned Brownian motion and the boundary limits of harmonic functions, Bull. Soc. Math. France 85 (1957), 431-458.

3. G. A. Hunt and R. L. Wheeden, Positive harmonic functions on Lipschitz domains, Trans. Amer. Math. Soc. 132 (1970), 307-322.

4. N. Ikeda and S. Watanabe, Stochastic differential equations and diffusion processes, NorthHolland, Amsterdam, 1981.

5. H. Kesten, Positive harmonic functions with zero boundary values, Proc. Sympos. Pure Math., vol. 35, part 1, Amer. Math. Soc., Providence, RI, 1979, pp. 349-352.

6. R. G. Pinsky, A probabilistic approach to a theorem of Gilbarg and Serrin, Israel J. Math. 74 (1991), 1-12.

7. set, Ann. Probab. 21 (1993), 453-481.

8. __ Positive harmonic functions and diffusion, Cambridge Tracts in Mathematics, Cambridge Univ. Press (to appear).

9. H. Aikawa, On the Martin boundary of Lipschitz strips, J. Math. Soc. Japan 38 (1986), 527-541.

Department of Mathematics, Northwestern University, Evanston, Illinois 60208

E-mail address: iof fe@math.nwu.edu

Technion-IsRael Institute of Technology, Haifa, IsRael

E-mail address: MAR4444@technion.technion.ac.il 